\section{Merck wins first PD1 sprint in US}

US regulators approved Merck \& Co.'s breakthrough cancer immunotherapy pembrolizumab, just over 3 years after it entered the clinic.

The lowdown: After a brief but bright surge through the clinic, the first of the much hyped and hoped-for programmed cell death protein 1

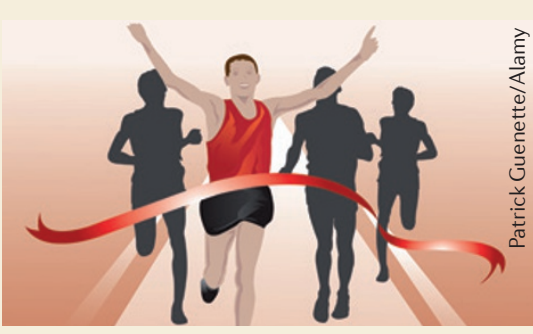

(PD1) inhibitors has reached the US market. These immunotherapies, like Bristol-Myers Squibb's ground-breaking CTLA4 (cytotoxic T-lymphocyte protein 4) inhibitor ipilimumab that came before them, relieve the brakes on T cells to engage and kill otherwise elusive tumour cells (Nature Rev. Drug Discov. 12, 489-492; 2013). On 4 September this year, the US Food and Drug Administration (FDA) approved Merck's pembrolizumab for patients with advanced or unresectable melanoma who no longer respond to other drugs. The drug was approved largely on the basis of a 173-patient, uncontrolled Phase I trial. Pembrolizumab shrank tumours in around $24 \%$ of patients. The effect lasted at least $1.4-8.5$ months.

But with some analysts having forecast a US\$30-billion market for immunotherapies like pembrolizumab, the race is far from over. For one thing, Merck's first-in-class approval is in a narrow indication with a burgeoning set of treatment options. The company is working towards other indications, including non-small-cell lung cancer (NSCLC). Bristol-Myers Squibb, another leader in the field, received approval in Japan in July this year for its PD1 inhibitor nivolumab for unresectable melanoma and plans to submit for US approval shortly. The company has also started a rolling submission in the US for nivolumab in NSCLC, and the drug is in Phase III trials for renal cell cancer. Roche and AstraZeneca, who are developing programmed cell death ligand 1 (PDL1) inhibitors, have also reached Phase III trials for NSCLC. The ultimate utility of the class moreover, is likely to turn on the success of combination trials. PD1 inhibitors and PDL1 inhibitors are being combined with a range of other agents, including other immunotherapeutics such as ipilimumab and inhibitors of the 41BB ligand receptor (also known as CD137).

While clinicians await more detailed clinical trial data, drug developers will also be watching the courts: on the same day that the FDA approved pembrolizumab, Bristol-Myers Squibb and its partner Ono Pharmaceutical sued Merck for patent portfolio violation. Merck says the lawsuit is without merit.

\section{Illuminating interleukin-5 data}

GlaxoSmithKline's mepolizumab succeeded in two Phase III asthma trials, whereas AstraZeneca's benralizumab failed in a Phase II chronic obstructive pulmonary disease trial. The lowdown: In 2000, GlaxoSmithKline (GSK) hit a wall when its interleukin-5 (IL-5)-specific antibody mepolizumab failed in its first Phase II trial. Nearly 15 years on, after a rethink on asthma trials (Nature Rev. Drug Discov. 11. 737-738; 2012) and increased insight into eosinophil function (Nature Rev. Drug Discov. 12.117-129; 2013), the company has found a way to the other side. The drug met its primary end point in a Phase III trial in 576 patients with severe eosinophilic asthma, reducing the rate of asthmatic exacerbations by $47 \%-53 \%$ compared with placebo, GSK recently reported (Ortega, H. G. et al. N. Engl.). Med. 8 Sep 2014). It also met its primary end point in a smaller 135-patient Phase III trial, reducing the daily oral glucocorticoid dose needed to effectively treat patients' symptoms (Bel, E. H. et al. N. Engl. J.Med, 8 Sep 2014).

A regulatory filing is now expected by the end of the year. Yet, a linked editorial notes that although the drug was safe and effective, "these studies do not suggest that all patients with uncontrolled asthma who have peripheral blood eosinophilia will require an expensive anti-IL-5 therapy for clinical benefit".

Parameswaran Nair, of McMaster University, Canada, points out that the exacerbation rate in the larger trial fell by over $50 \%$ even with just the placebo, which suggests that many patients may benefit just from improved adherence to available drug regimens and good clinical practice (Nair. P. N. Engl.J. Med. 8 Sep 2014).

AstraZeneca's IL-5 receptor (IL-5R)-specific benralizumab and Teva's IL-5-specific reslizumab are also in Phase III trials for asthma.

AstraZeneca and GSK have also both moved their drugs into Phase III for chronic obstructive pulmonary disease (COPD). AstraZeneca reported in September, however, that their benralizumab failed to reduce the exacerbation rate in an 82-patient Phase II trial (Lancet Respir. Med. 8 Sep 2014). "The results of prespecified subgroup analysis support further investigation of benralizumab in patients with COPD and eosinophilia," the authors note.

\section{Reanalyse this}

Re-evaluations of clinical trial results are rare, but they often point to new conclusions. The lowdown: With industry and academic institutions continuing to negotiate the terms of increased clinical trial result transparency, John loannidis of Stanford University and his colleagues scoured the literature to find out how often clinical trial results are reanalysed, and what these reanalyses yield. Searching Medline from its inception until March 2014, they identified only 37 reanalyses of clinical trial results (JAMA 312, 1024-1032; 2014). Thirteen of these reanalyses (35\%) led to an interpretation different from that of the original article, including three (8\%) that showed that different patients should be treated, one (3\%) that showed that fewer patients should be treated, and nine (24\%) that showed that more patients should be treated. Reanalyses differed most commonly in terms of the statistical or analytical approaches that were used and in definitions or measurements of the outcome of interest.

Only five of the reanalyses were done by a totally independent group of researchers, the analysis also found. "Involving authors of the original article in reanalyses may be a condition for providing access to data and may ensure that direct knowledge of study nuances is accounted for in a reanalysis. Involving such authors might also limit the independence of any coauthors to refute initial results if the original authors have commitments to their findings," the authors write.

In a linked editorial, Yale University's Harlan Krumholz and a co-author call the findings "surprising and discomforting" (JAMA 312. 1002-1003; 2014). The fact that reanalyses often point to new conclusions fuels calls for even more transparency and openness with clinical trial data, they add. "Replication is a vital part of the scientific method... The recognition that one trial can potentially lead to different findings and conclusions depending on many discretionary decisions that are made about the data and reanalyses almost mandates that those choices are transparent and described in detail — and that others have the chance to replicate them." 